\title{
Needle Detection and Tracking in the TIPS Endovascular Procedure
}

\author{
Benoît Jolly, Mark Van Horn, Stephen Aylward, and Elizabeth Bullitt \\ MIDAG, University of North Carolina, Chapel Hill, NC 27599 \\ http://casilab.med.unc.edu/needletrack
}

\begin{abstract}
The TIPS procedure is an endovascular operation guided by biplane fluoroscopy. The goal is to pass a needle from the hepatic vein through the liver into the portal vein. We are developing a software for real-time, 3D guidance of the procedure. This paper discusses means of automatically defining the needle from biplane fluoroscopic views.
\end{abstract}

\section{Introduction}

We aim to provide a method of guiding endovascular operations by 1) segmenting vessels from preoperative 3D images, 2) registering 3D vessels with their projections as seen by biplane fluoroscopy, 3) extracting the needle from fluoroscopic views, and 4) reconstructing the needle into $3 \mathrm{D}$. This paper discusses methods of automatically defining a needle from fluoroscopic images.
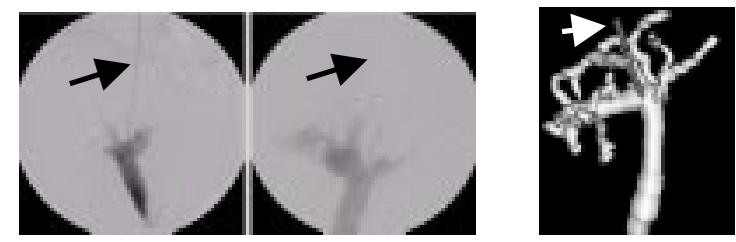

Fig. 1. Fluoroscopic biplane views of the portal vein (bottom dark tree) and the needle (top gray tube) and (right) the needle reconstructed into 3D from the fluoroscopic views and shown relative to the $3 \mathrm{D}$ segmentation of the portal vein. The arrow points to the needle.

\section{Method}

Existing methods of curve tracking, like the Hough transform or the Polar transform, track straight valleys or defined curves [1,2]. The problem is that there are no a priori values for the needle curvature. The method described here is somewhat similar to that described by Aylward in defining 3D curves [3]. The process begins from an initial seed point that can be defined either manually or automatically. The needle is viewed as an intensity valley that can be tracked segment by segment by searching in the tangent direction for the next needle point, with the initial segment defined using the prior knowledge that the needle tends to pass rostro-caudally. We convolve a 
second derivative of a Gaussian orthogonally on the area to find a possible location of a valley. The 3 best filter responses represent possible positions of the next point.

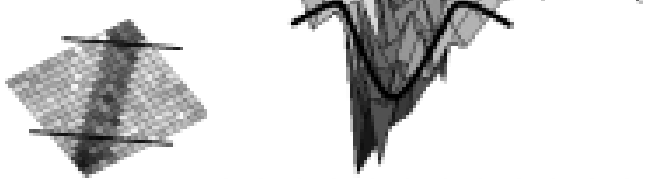

Fig. 2. Left: Fluoroscopic view of the needle. A step has been taken in the tangent direction and the thin horizontal lines represent the filtering lines. Right: Height map of intensity values and the filters applied (black).

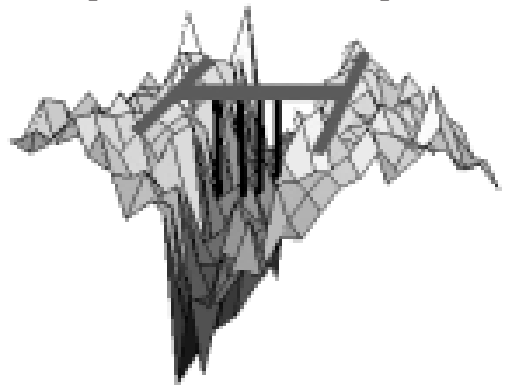

Fig. 3. Model used to quantify the valley depth

We use an $\mathrm{H}$-shaped model (fig 3) to quantify the valley. Its parallel branches give an average of the environment of the needle. Its center is then compared to the intensity value in the valley to get the depth. The path is selected that best defines an intensity valley on the image data and the process then iterates until no clear valley point can be defined (the needle tip).

\section{Results}

We have tested the algorithm on needle passes for 4 patients undergoing the TIPS procedure. Eight thousand images were used to test needle tracking given an initial needle definition. The tracking was stable in every case without drift. The computation time is $60 \mathrm{~ms}$ for a long needle inserted through the liver. A second test using a randomly selected AP and lateral image from each patient noted a problem in only a single case, resulting from a second wire that crossed the needle on one projection. There may be an easy solution to this problem by asking the nurses to place any outside wires to avoid overlap with the region of interest on projection.

\section{References}

1. D. Vernon Machine Vision, Prentice-Hall, 1991, Chap. 6.

2. E. Gamma, R. Helm, R. Johnson, J. Vlissides, "Design Patterns", Addison Wesley, 1995.

3. Aylward S, Bullitt E (2002) Initialization, noise, singularities and scale in height ridge traversal for tubular object centerline extraction. IEEE-TMI 21:61-75. 